\title{
Posttraumatic Stress Disorder and Alterations in Resting Heart Rate Variability: A Systematic Review and Meta-Analysis
}

\author{
Fenfen $\mathrm{Ge}^{1}$, Minlan Yuan ${ }^{1}$, Ying $\mathrm{Li}^{2}$, and Wei Zhang ${ }^{1 凶}$ \\ ${ }^{1}$ Mental Health Center of West China Hospital, Sichuan University, Chengdu, China \\ ${ }^{2}$ Embedded System and Intelligent Computing Laboratory, University of Electronic Science and Technology of China, Chengdu, China
}

Objective The functions of both the central and peripheral autonomic nervous system, indexed by heart rate variability (HRV), are affected by psychology and physiology. This review summarizes the results of studies comparing the evaluation of HRV parameters between individuals with posttraumatic stress disorder (PTSD) and healthy controls.

Methods Eligible studies $(\mathrm{n}=499)$ were identified through literature searches of the EMBASE, Medline, PubMed and Web of Science databases. Nineteen studies met our inclusion criteria. A random effects model was used, and standardized mean differences for highfrequency HRV(HF-HRV), low-frequency HRV(LF-HRV) and the root mean square of successive R-R interval differences (RMSSD) were calculated.

Results Significant effects were found for HF-HRV [p $<0.0001, \mathrm{Z}=4.18$; Hedges' $=-1.58,95 \%$ confidence interval (CI) $(-2.32,-0.84)$; $\mathrm{k}=14]$ and RMSSD [p<0.00001, $\mathrm{Z}=4.80$; Hedges' $=-1.96,95 \% \mathrm{CI}(-2.76,-1.16) ; \mathrm{k}=9]$ relative to healthy controls. Considerable heterogeneity was revealed, but the main effects for HF-HRV and RMSSD were robust in subsequent meta-regression and subgroup analyses.

Conclusion Given the relationships among low vagal state, inflammation, and alterations in brain structure and function, including executive function and emotion regulation, reduced HRV may be regarded as an endophenotype in PTSD research. Psychiatry Investig 2020;17(1):9-20

Key Words Heart rate variability, Posttraumatic stress disorder, Endophenotype, Meta-analysis.

\section{INTRODUCTION}

Direct or indirect exposure to actual or threatened death (e.g., via road traffic accidents, natural disasters, witnessing the death of others) or severe traumatic events (e.g., sexual violence/abuse, combat) may lead to posttraumatic stress disorder (PTSD). PTSD affects approximately $6.8-12 \%$ of the US adult population at some point in their lifetime. ${ }^{1}$ Symptoms of PTSD (e.g. intrusive memories, re-experience and avoidance) have negative impacts on both physical (e.g., digestive, respiratory and cardiovascular diseases) $)^{2,3}$ and psychological (e.g., negative mood, recklessness, substance abuse and comorbidity with other mental illnesses) ${ }^{4}$ characteristics. PTSD not only affects quality of life ${ }^{5}$ but also increases the risk of suicide. ${ }^{6}$ Thus,

Received: May 7, 2019 Revised: October 6, 2019

Accepted: October 14, 2019

$\square$ Correspondence: Wei Zhang, $\mathrm{PhD}$

Mental Health Center of West China Hospital, Sichuan University, No.37 Guoxue Alley, Wuhou District, Chengdu City, 610041 Sichuan Province, PR China

Tel: +18980601010, Fax: +028-85582944, E-mail: weizhang27@163.com

(a) This is an Open Access article distributed under the terms of the Creative Commons Attribution Non-Commercial License (https://creativecommons.org/licenses/bync/4.0) which permits unrestricted non-commercial use, distribution, and reproduction in any medium, provided the original work is properly cited.
PTSD is a serious public health problem that places heavy burdens on both individuals and society. However, PTSD is often not diagnosed and treated in a timely manner due to the overlapping symptoms of other psychiatric disorders and the tendency of clinicians to inadequately examine for symptoms of traumatic stress during clinical practice. ${ }^{7}$

The autonomic nervous system (ANS), which includes the sympathetic nervous system (SNS) and the parasympathetic nervous system (PNS), plays an important role in the onset and progression of PTSD. ${ }^{8}$ The SNS is considered to offer the most immediate response to a stressor via the reaction of the sympatho-adrenomedullary arm reacting. ${ }^{9}$ Then, the activation of the ANS declines quickly results in short-lived response, as the PNS is activated and acetylcholine is released into the system after the immediate stress events have passed. Thus, when the acute stressors experienced by individuals are converted to chronic or recurrent stress, the SNS system may be continuously activated without the confrontation of the PNS. Sustained hyperactivity of the SNS and abnormal of the PNS is accompanied by increasing catecholamine (epinephrine and norepinephrine) levels and decreasing acetylcholine in individuals with PTSD..$^{10}$ The dysregulation of the 
ANS may link the physiological and psychological pathologies of PTSD. ${ }^{11}$ Heart rate variability (HRV) is the most commonly used measure of ANS activity because it is noninvasive as well as easy and inexpensive to monitor. ${ }^{12}$

Metrics of HRV are widely used in the field of behavioral and biomedical sciences. In 1996, the European Society for Cardiology and the North American Society of Pacing and Electrophysiology formally defined HRV as "heart rate oscillations within the beat-to-beat variability." ${ }^{13}$ Since then, an increasing number of studies have focused on short-term (minutes to $1 \mathrm{~h}$ ) or long-term (24 h) HRV. ${ }^{14}$ HRV measures the state of the ANS based on the notion of balance between the ANS and SNS. Variability is considered a proxy for the health of a system, and decreased variability being a sign of a pathological condition. ${ }^{15}$ And HRV indices can be used to better understand the peripheral physiological correlates of brain and behavioral processes (e.g., emotion and cognition), ${ }^{16}$ possibly by reflecting the functionality of the prefrontal cortex. ${ }^{17} \mathrm{HRV}$ indices include the following commonly used indices: low-frequency power (LF, $0.01-0.15 \mathrm{~Hz}$ ) and high-frequency power (HF, $0.15-0.40 \mathrm{~Hz}$ ) in both $\mathrm{ms}^{2}$ and normalized units, the ratio of LF to HF (LF/HF), the standard deviation of normal-tonormal $(\mathrm{NN})$ intervals $(\mathrm{SDNN})$ and the root mean square of successive differences between NN intervals (RMSSD). LF and $\mathrm{HF}$ are frequency domain indices, and SDNN and RMSSD are time domain (TD) indices. HF-HRV is usually considered a proxy for the PNS state and is similar to respiratory sinus arrhythmia (RSA). ${ }^{18}$ LF-HRV is regulated by the SNS and PNS, and high LF reflects increased SNS activity. ${ }^{19}$ The ratio of LF/ HF might reflect the vagal balance. However, recent studies have suggested that the LF/HF ratio does not have a mathematical basis and is not an appropriate index of sympathovagal balance. ${ }^{20}$ Among time domain metrics, the RMSSD has been thought to be the most appropriate method for quantifying HRV, and it closely represents PNS. ${ }^{21,22}$

Studies show that PTSD is associated with HRV. For example, the risk of cardiovascular disease is increased in PTSD. ${ }^{23}$ HRV is a reliable index of this risk disease. ${ }^{24}$ Dysfunction of emotion is associated with HRV. ${ }^{25-27}$ Moreover, some brain regions (e.g., anterior cingulate cortex and medial prefrontal cortex) with abnormalities are associated with HRV. ${ }^{28}$ However, findings regarding the alterations in HRV in patients with PTSD are inconsistent. Regarding TD, research has revealed that PTSD may be related to decreased RMSSD and SDNN, ${ }^{29,30}$ although not consistently so. ${ }^{31}$ There is some evidence suggesting that HF is lower among individuals with PTSD; ${ }^{32,33}$ however, some studies have not reported this effect, ${ }^{34}$ and others have only reported TD and not frequency domain indices. ${ }^{31}$ Some studies have reported elevated LF in individuals with PTSD, ${ }^{30,35}$ al- though inconsistent results have been reported. ${ }^{36,37}$ The earliest review of the relationship between PTSD and HRV was published in 2007 by Friedman. ${ }^{38}$ A subsequent 2014 meta-analysis of HRV alterations with anxiety found that HF-HRV was reduced in PTSD patients relative to healthy controls, whereas LF-HRV did not differ significantly. ${ }^{39} \mathrm{~A}$ limitation of the above studies is that they did not consider potential confounding factors of HRV in PTSD patients. Given the importance of increasing statistical power and reducing false positive rates, an up-to-date meta-analysis of studies of HRV and PTSD is needed.

We aimed to summarize the current studies comparing HRV between PTSD patients and healthy controls. We concentrated on quantifying the frequency domain and TD HRV indices. Furthermore, we investigated the source of heterogeneity (e.g. clinical, methodological), and analyzed potential variables via meta-regression.

\section{METHODS}

\section{Search strategy}

The meta-analysis was conducted according to the PRISMA guidelines and registered in the PROSPERO database (registration number CRD42018099682). At the time of registration, no similar reviews were listed in the PROSPERO database. Four databases (PubMed, EMBASE, Medline, and Web of Science) were searched for the terms "heart rate variability," "HRV," "vagal," "automatic nervous system," "ANS," "posttraumatic stress disorder," "PTSD," and "post-traumatic stress disorder." Articles were also obtained by reviewing cited references. We retrieved on those articles published after 1996 as the guidelines for HRV testing and calculations were published in 1996. The article search was completed in August 2018. The article inclusion criteria were as follows: 1 ) a definitive diagnosis of PTSD as defined by the Diagnostic and Statistical Manual of Mental Disorders (DSM-III), DSM-IV, DSM-5 or International Classification of Diseases (ICD-10) and comparisons with a healthy control group without traumatic events and psychiatric disorder; 2) HRV index recording and calculation in accordance with the guidelines of the Task Force of the European Society of Cardiology and the North American Society of Pacing and Electrophysiology; 3) resting state HRV reported; and 4) study published in English. Two authors independently reviewed all potentially relevant manuscripts, and cases of disagreement were resolved via consultation with the third investigator.

\section{Data extraction}

Using only the frequency domain or time domain HRV to assess the patients with PTSD has limitations, ${ }^{40}$ so frequency 
domain and time domain measures were included in this meta-analysis. If more than one TD measure was reported in a study, RMSSD was prioritized, followed by SDNN. We chose to report only RMSSD in this review because RMSSD and SDNN offer identical information about the HRV and the former is more stable. ${ }^{41}$ Regarding the frequency domain, HF-HRV and LF-HRV measures are the most commonly reported measures in the PTSD literature. Thus, the frequency domain measures extracted in this study were HF-HRV and LF-HRV. If the data were presented only in graphs in an article, we obtained the original data via email with the corresponding author. Where longitudinal data were reported, we extracted only the baseline data to reduce the influences of confounding factors. If $\mathrm{HRV}$ parameters were reported separately for day and night in a study, we extracted only those for night. Nighttime HRV is more stable than daytime HRV in reflecting the activity of the vagal nerve due to less body movement and fewer confounding factors. For the meta-regression and subgroup analyses, the following data were also extracted: year of publication, country, mean participant age, quality score of the study, the score of the depression scale, proportion of males, source of participants, recording duration and recording values.

\section{Quality assessment}

In 2009, Tak et al. ${ }^{42}$ developed a scale to assess HRV in methodological studies. The instrument evaluates study quality based on the following three dimensions: appropriate selection of participants, appropriate quantification of HRV and appropriate control for confounders. Each dimension includes three questions. The total score of this scale ranges from 0 to 18 points, with a higher score indicating higher quality.

\section{Meta-analysis statistics}

\section{Effect size estimation}

Meta-analytic computations were performed using Review Manager 5.1 (https://community.cochrane.org/help/toolsand-software/revman-5). ${ }^{43} \mathrm{~A}$ random effects (RE) model was used in this study. To investigate the magnitude of the effect size of each variable across the different studies, we calculated the standardized mean difference (SMD, excluding the influence of the unit). The significance of Hedges' $\mathrm{g}$ was determined using 95\% confidence intervals (95\% CIs). When the $95 \%$ CI did not overlap the null hypothesis value of zero, the group difference was considered significant at the $\alpha=0.05$ level. In one study, only the maximum and minimum values were reported. ${ }^{44}$ We calculated the standard deviation according to the Cochrane guide. ${ }^{43}$ Heterogeneity was assessed using $\mathrm{I}^{2}$ values, with $\mathrm{I}^{2} \geq 25 \%, \mathrm{I}^{2} \geq 50 \%$, and $\mathrm{I}^{2} \geq 75 \%$ interpreted as low, medium and high heterogeneity, respectively. ${ }^{45}$

\section{Meta-regression and subgroup analyses}

Regression analysis and subgroup analysis were performed using Stata 15.0 (StataCrop LP, College Station, TX, USA) and Review Manager 5.1 (https://community.cochrane.org/help/ tools-and-software/revman-5), respectively. When significant heterogeneity was observed $\left(\mathrm{I}^{2} \geq 50 \%\right)$, we use mixed mode regression analysis and the restricted maximum-likelihood estimation (RMLE) method to explore the source of heterogeneity among studies. The potential variables investigated were year of publication, mean participant age, study quality score, the score of depression scale and the proportion of male participants. Most studies use the Beck Depression Scale (BDI) to assess depression, Ray's ${ }^{46}$ study use the Patient Health Questionnaire (PHQ-9), and Cohen's ${ }^{33}$ study used the Hamilton Depression Scale (HAMD). Some of the variables of interest were limited to a small number of studies $(n<10)$; in these cases, subgroup analyses were conducted. These variables included the country of study (Asia, Europe and America), source of participants (clinical, community and clinical \& community), recording duration (short term and long term) and recording values (raw and log-transformed). To explore whether the effects of the HRV indices in PTSD patients were epiphenomenal to medication use (e.g., use of antidepressants, benzodiazepines, or antipsychotics), we analyzed studies that excluded current medicine users.

\section{RESULTS}

\section{Inclusion of studies}

According to the search terms, 499 articles were compiled from the four databases. Among these, 206 duplicates, 3 case reports, and 12 reviews were excluded. After reading the titles and abstracts, the remaining 47 articles were potentially eligible for inclusion. After reading the full texts of these 47 articles, 19 articles were finally included (Figure 1).

\section{Study and sample characteristics}

The characteristics of the 19 studies that compared resting state HF, LF, and TD HRV measures between participants who met the inclusion criteria are summarized in Table 1. Of the 19 studies included, 15 reported HF, 12 reported LF, and 10 studies reported RMSSD. 16 studies compared HRV between 2 groups (PTSD vs health controls without traumatic events) and 4 studies ${ }^{35,36,47,48}$ compared HRV between 3 groups (PTSD vs. healthy controls without traumatic events and healthy controls with traumatic events). 


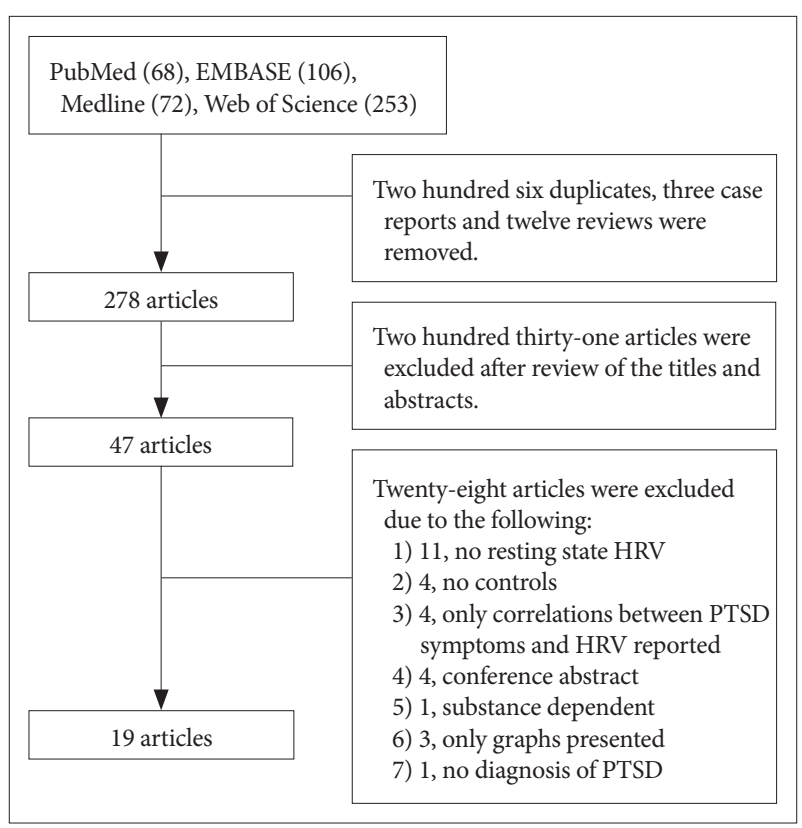

Figure 1. Systematic search of the literature: PRISMA flowchart. PTSD: posttraumatic stress disorder, HRV: heart rate variability.

\section{Main effects}

The meta-analysis revealed a significant effect of PTSD status on HF-HRV ( $\mathrm{Z}=4.18, \mathrm{p}<0.0001)$, with individuals with PTSD ( $\mathrm{n}=435)$ having lower HF-HRV than healthy controls $\left[\mathrm{n}=940 ; \mathrm{g}=-1.58(-2.32,-0.84) ; \mathrm{I}^{2}=96 \% ; \mathrm{k}=14\right]$. Figure 2 shows the HF-HRV results. Visual inspection of the funnel plot demonstrates only slight asymmetry, suggesting that nonsignificant studies were lacking (Figure 3). Next, to assess possible bias, we calculated the main effect after excluding extreme SMD values (i.e., $>2$ ) and still found a significant effect $\left[\mathrm{Z}=2.20, \mathrm{p}=0.03 ; \mathrm{g}=-0.31(-0.59,-0.33) ; \mathrm{I}^{2}=67 \% ; \mathrm{k}=10\right]$. We also calculated the effect omitting the study that only reported RSA. Again, a significant effect was found $[\mathrm{Z}=4.05$, $\mathrm{p}<0.0001 ; \mathrm{g}=-1.65(-2.45,-0.85) ; \mathrm{I}^{2}=96 \% ; \mathrm{k}=13$ ]. To explore whether the reduction in HRV in individuals with PTSD relative to controls was affected by medication use (including antidepressants, benzodiazepines, or antipsychotics), we repeated the calculation excluding medication users. There was a significant decrease in HF-HRV among unmedicated PTSD patients relative to unmedicated healthy controls $\left[\mathrm{Z}=2.59, \mathrm{p}=0.010 ; \mathrm{g}=-1.60(-2.82,-0.39) ; \mathrm{I}^{2}=96 \% ; \mathrm{k}=5\right]$.

The meta-analysis with LF-HRV as the dependent variable did not reveal a significant effect of PTSD status $(\mathrm{Z}=1.42$, $\mathrm{p}=0.15$ ), indicating that there was no significant difference in LF between individuals with PTSD $(n=403)$ and healthy controls $\left[\mathrm{n}=915 ; \mathrm{g}=-0.63(-1.49,0.24) ; \mathrm{I}^{2}=97 \% ; \mathrm{k}=12\right]$. We then calculated the main effect after excluding extreme SMD values (i.e., >2) and again found no significant effect $[\mathrm{Z}=1.97$, $\left.\mathrm{p}=0.05 ; \mathrm{g}=-0.28(-0.56,-0.00) ; \mathrm{I}^{2}=64 \% ; \mathrm{k}=8\right]$. Five of thirteen studies excluded medication users. Among these unmedicated PTSD patients $(n=161)$ and healthy controls $(n=329)$, there was no significant effect of PTSD status on LF-HRV $\left[\mathrm{Z}=0.28, \mathrm{p}=0.78 ; \mathrm{g}=0.32(-1.97,2.61) ; \mathrm{I}^{2}=98 \% ; \mathrm{k}=4\right]$.

When RMSSD-HRV was included as the dependent variable, a significant effect of PTSD status was observed $(\mathrm{Z}=4.80$, $\mathrm{p}<0.00001)$, with PTSD patients $(\mathrm{n}=304)$ having lower RMSSD than healthy controls $[n=325 ; g=-1.96(-2.76,-1.16)$; $\left.\mathrm{I}^{2}=94 \% ; \mathrm{k}=9\right]$. The details of each study and SMD are shown in Figure 4. Visual observation of the funnel plot (Figure 5) reveals a lack of data in the lower right of the plot. We then calculated the effect of PTSD status after excluding studies with extreme SMD values (i.e., $>2$ ); a significant effect was found $\left[\mathrm{Z}=5.51, \mathrm{p}<0.00001 ; \mathrm{g}=-0.89(-1.20,-0.57) ; \mathrm{I}^{2}=42 \% ; \mathrm{k}=5\right]$. After excluding studies that did not report standard deviations, a significant effect was again found, suggesting that the declined vagal activity in PTSD was robust $[\mathrm{Z}=4.30, \mathrm{p}<0.0001 ; \mathrm{g}=-1.80$ $\left.(-2.62,-0.98) ; \mathrm{I}^{2}=94 \% ; \mathrm{k}=8\right]$. Four of ten studies excluded medication users. Among the unmedicated PTSD patients $(n=77)$ and healthy controls ( $\mathrm{n}=82)$, a significant effect of PTSD status on RMSSD-HRV was observed [ $\mathrm{Z}=3.51, \mathrm{p}=0.0005$; $\mathrm{g}=-1.44$ $\left.(-2.24,-0.64) ; I^{2}=74 \% ; \mathrm{k}=3\right]$.

The results of the meta-regression are presented in Table 2. Year of publication, study quality score, mean participant age and proportion of males did not show significant heterogeneity $(p>0.05)$. The results of the subgroup analyses are shown in Table 3. When we evaluated the effects of PTSD status for different countries separately, and varied results were obtained. A significant effect of PTSD status on RMSSD-HRV was found for three country groups; however, the effect on HF-HRV was not significant for studies in America. When evaluating the effects for different sources of participants (clinical, community and clinical \& community), a significant effect of PTSD status on RMSSD-HRV was observed for all three groups; however, the effect in the clinical populations was large, whereas that in the community populations was medium. The effect of PTSD status on HF-HRV was significant for the clinical populations but not the community and clinical \& community populations. The calculation of methodological heterogeneity demonstrated that all but one HRV indices subgroup demonstrated a significant effect. The PTSD participants showed lower HRV indices than did healthy controls regardless of the applied methods except in the case of HF-HRV, where no significant effect of PTSD status among studies applying long-time recording was found.

\section{DISCUSSION}

This meta-analysis analyzed existing studies on HRV in PTSD. When we investigated the HRV indices, we found re- 


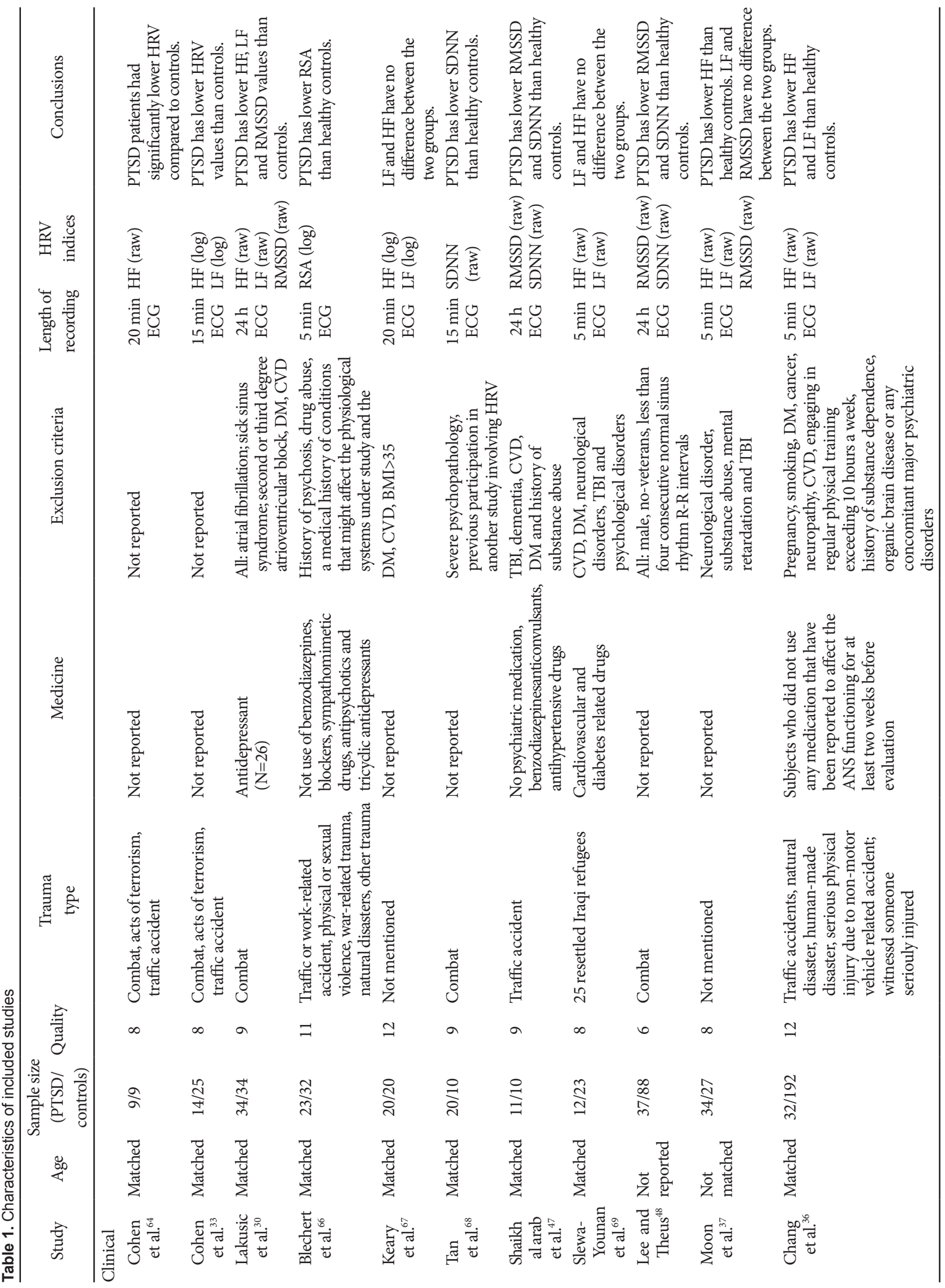




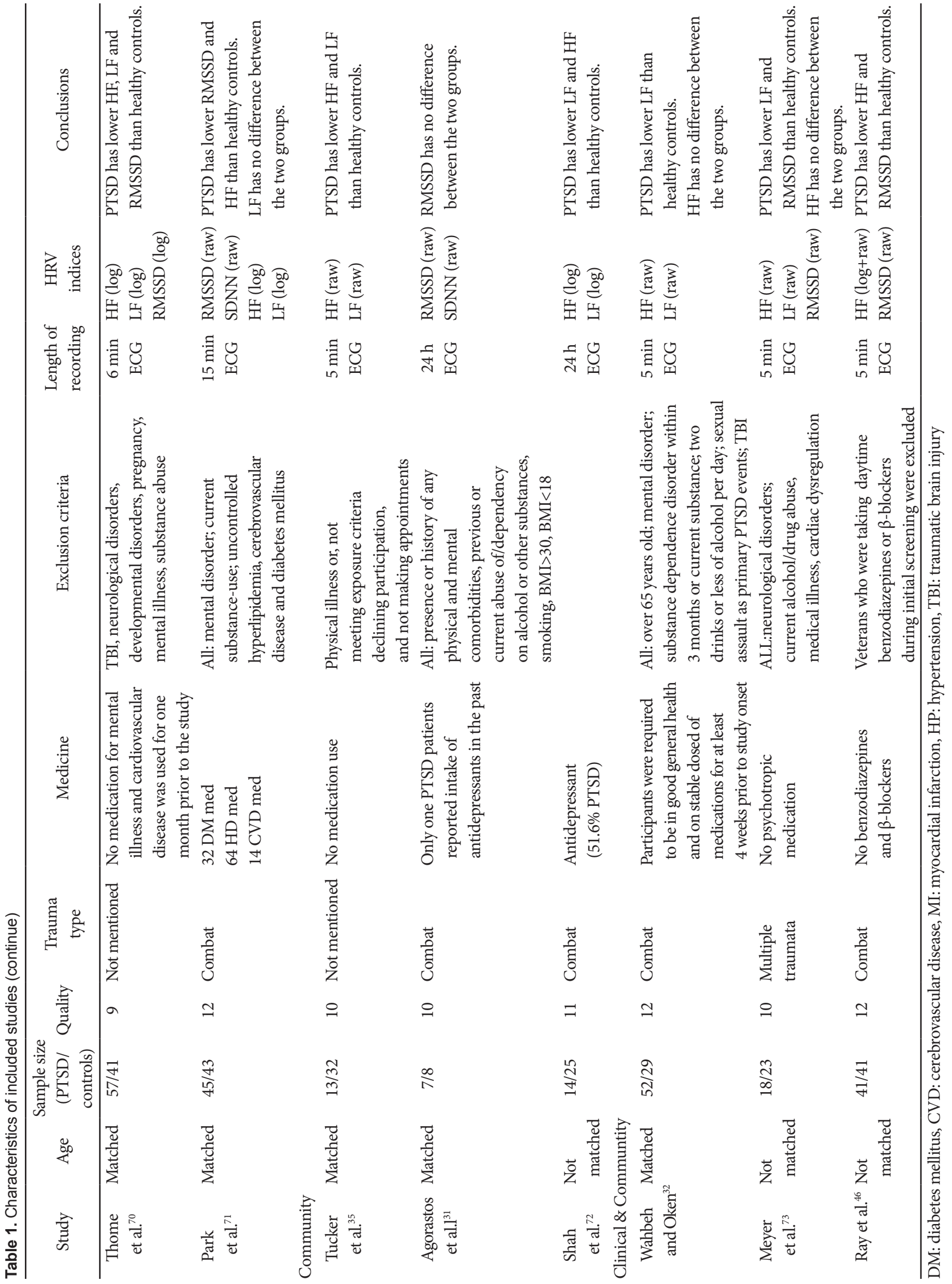




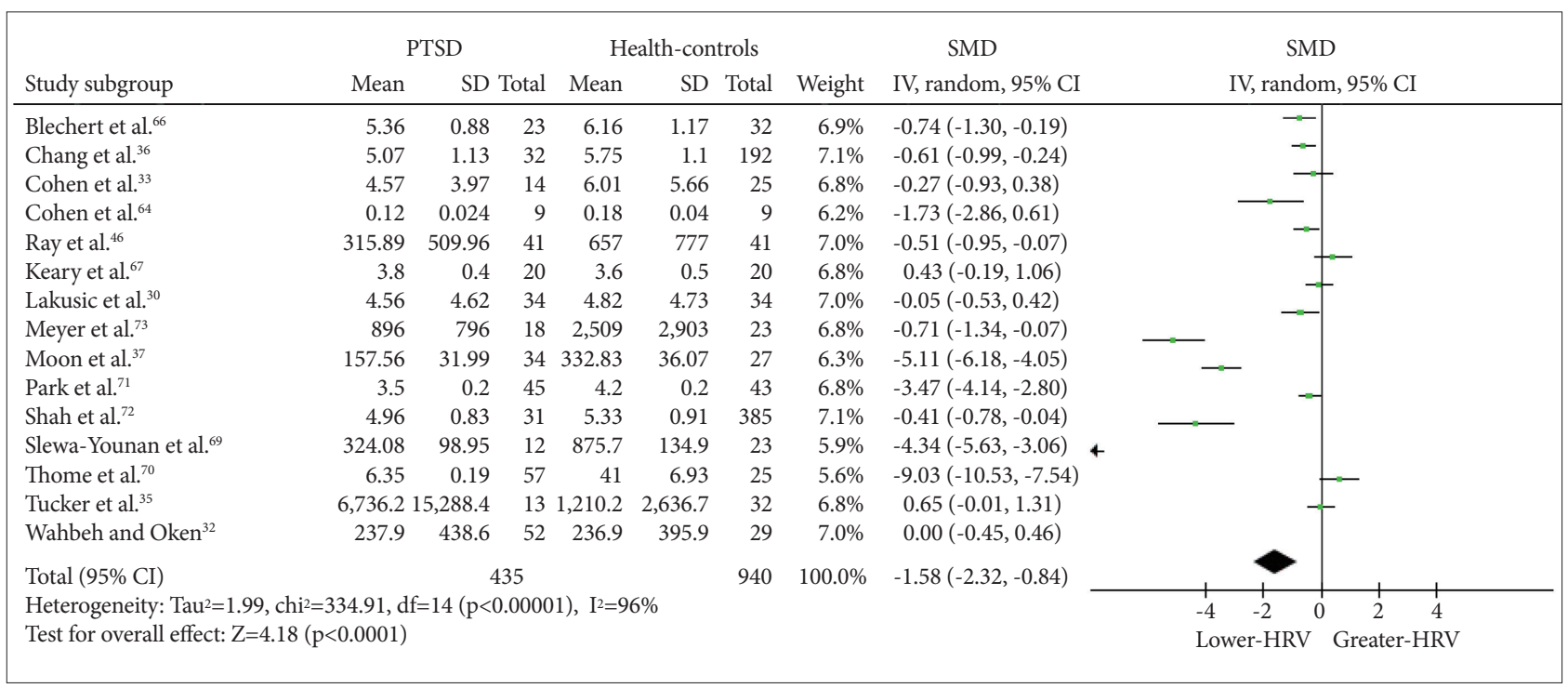

Figure 2. SMD in HF-HRV between individuals with PTSD and healthy controls. SMD: standard mean difference, HF-HRV: high-frequency heart rate variability, PTSD: posttraumatic stress disorder.

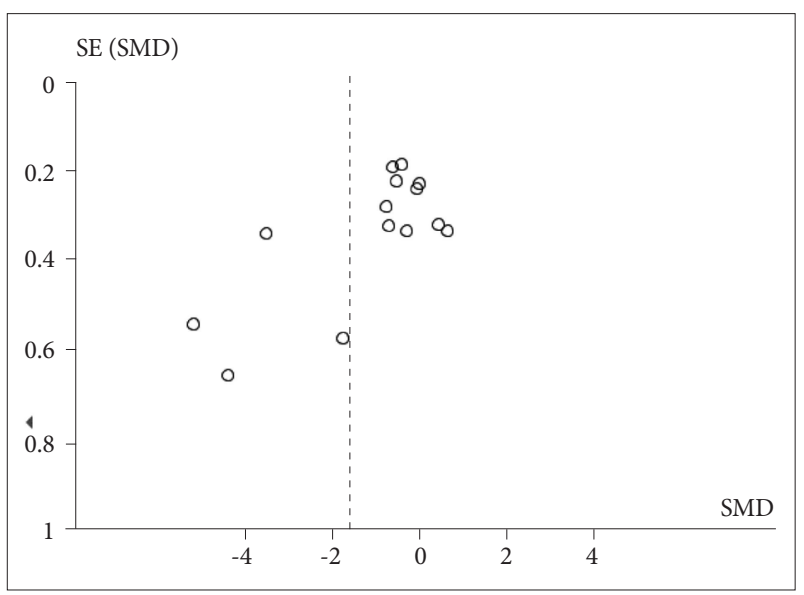

Figure 3. Meta-analysis funnel-plot comparing the high-frequency heart rate variability in individuals with posttraumatic stress disorder and healthy controls. SMD: standard mean difference.

ductions in HF-HRV (but not LF-HRV) and RMSSD. This finding suggests that HRV alterations in PTSD are nuanced and that some indices of HRV have implications for the diagnosis and treatment of PTSD. To evaluate the robustness of our findings, we calculated differences in HRV in different subgroups, including the source of participants, methodology and medication-use subgroups. In the meta-regression analyses, there was no significant influence of the variables of interest on the resting HRV (HF-HRV and RMSSD) difference between the PTSD participants and healthy controls. The subgroup analyses revealed subgroup differences for HF-HRV: no significant reduction in HF-HRV in PTSD participants relative to controls was found in the American subgroup, in the community and clinical \& community subgroups, and in the long-term recording subgroup. Only one of the subgroup analyses re- vealed variation in subgroups in the difference for RMSSD: no significant decline in RMSSD in individuals with PTSD relative to healthy controls was observed in the community subgroup. This finding suggests that RMSSD has greater robustness than $\mathrm{HF}$ as an HRV measure. However, given the small number of studies evaluated, future research is needed to confirm the reliability of these findings. Among the studies excluding medicated individuals, individuals with PTSD had lower HF-HRV and RMSSD than healthy controls. Thus, it is unlikely that the reductions in HF and RMSSD in PTSD individuals can be fully attributed to the intake of antipsychotic or benzodiazepine medications.

\section{Effects of PTSD on the frequency and time domains}

We found reductions in HF-HRV and RMSSD in PTSD, with a more robust reduction in the latter being more robust. Generally, high HRV reflects sympathovagal balance or wellconditioned adaptability of the ANS, whereas low HRV is a sign of deficiency and abnormal regulation of the ANS. ${ }^{49}$ PTSD is associated with the withdrawal of parasympathetic cardiac control, which is typically observed in the HF domain. ${ }^{29}$ The current results are consistent with those of the Hauschildt study, ${ }^{29}$ which showed that a lower HF-HRV is significantly associated with PTSD. In TD analyses, RMSSD is used a measure of cardiac change and reflects the overall degree of ANS activity. ${ }^{50}$ The significantly reduced RMSSD in individuals with PTSD relative to that in healthy controls might also result from the decline in PNS function due to trauma events in PTSD individuals. The results are in accordance with the theory of neurovisceral integration, in which low PNS function is an indicator of impaired central-peripheral neural 


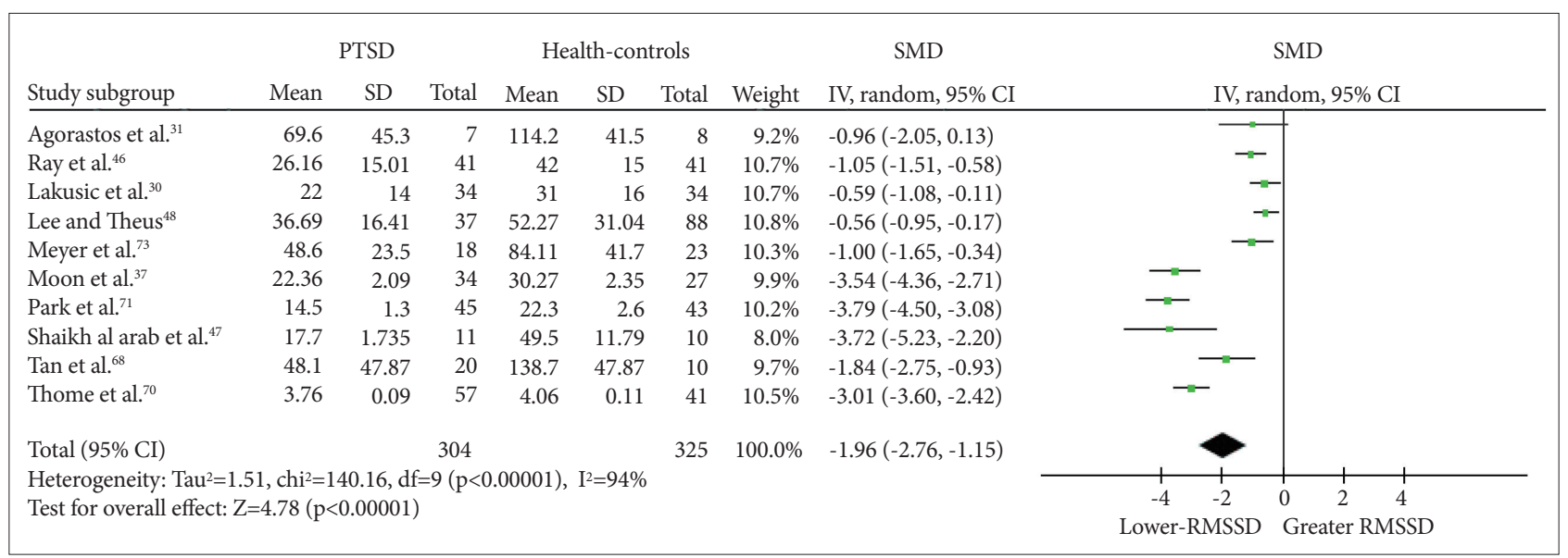

Figure 4. SMD in RMSSD-HRV between individuals with PTSD and healthy controls. PTSD: posttraumatic stress disorder, SMD: standard mean difference, RMSSD-HRV: root mean square of successive R-R interval differences-heart rate variability.

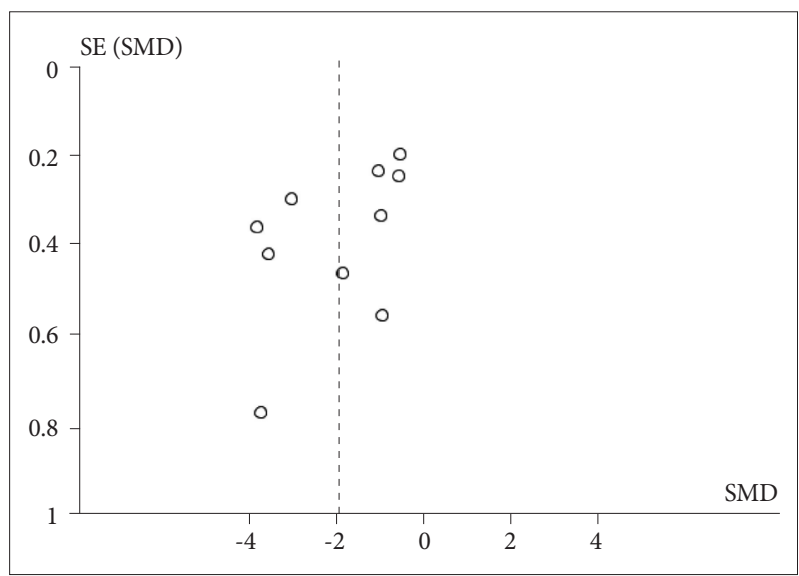

Figure 5. Meta-analysis funnel-plot comparing the root mean square of successive $R-R$ interval differences-heart rate variability in individuals with posttraumatic stress disorder and healthy controls. SMD: standard mean difference.

feedback. According to this theory, when an individual with low PNS function encounters environmental changes, he or she lacks sufficient psychophysiological resources to deal with them. ${ }^{27}$ Evidence suggests that individuals with high HRV at rest have higher scores on trait resilience psychometric scales. ${ }^{51}$ Individual with PTSD may continuously perceive threats and experience fear due to disinhibition of the threat circuits. ${ }^{52,53}$

The PNS can release acetylcholine to modulate the immune system and stimulate the cholinergic anti-inflammatory pathway to inhibit cytokine production. ${ }^{54,55}$ Accordingly, increased inflammation in patients with PTSD is observed. A recent meta-analysis reported that IL- $1 \beta$, IL-6, TNF- $\alpha$ and IFN- $\gamma$ levels were increased in patients with PTSD relative to healthy controls. ${ }^{56}$ In addition, neuroimaging studies have found that inflammation can alter brain structure and function. For example, IL-6 can modulate the connection between the amygdala and the anterior cingulate cortex (ACC). ${ }^{23}$ Individ- uals with increased inflammation showed a stronger connection between the amygdala and the dorsomedial prefrontal cortex (PFC) than did individuals with normal levels of inflammation. ${ }^{57}$ These regions, as well as the ACC and media prefrontal cortex have been found to be associated with HRV. ${ }^{28}$ Thus, a reduction of HRV can be regarded as an endophenotype for PTSD, characterized by increased inflammation and alterations in brain structure and function. HRV as an endophenotype could play an important role in the diagnosis and treatment of PTSD and serve as a valuable biomarker in research.

\section{Medicine use}

The present study suggests that the HRV decrease observed in PTSD is not completely attributable to medication use. In the studies of unmedicated individuals, we found that individuals with PTSD had lower HF-HRV and RMSSD than did healthy controls. In all of these studies, except one study that investigated drug-naive PTSD, ${ }^{36}$ the participants had received antidepressant treatment at some time prior to the assessment. The washout period in these studies was approximately 4 weeks, and it is possible that the previous medication continued to have influence on the ANS at the time of the study. Few studies reporting whether HRV indices in patients with PTSD are influenced by medication, and their results are inconsistent. Cohen reported that HRV indices in patients with PTSD returned to normal after treatment with selective serotonin reuptake inhibitors (SSRIs). ${ }^{58}$ However, other studies found that SSRIs do not improve HRV reductions in individuals with depressive symptoms. ${ }^{59} \mathrm{~A}$ review reported high dropout rates in SSRI trials and in trials with patients with PTSD. ${ }^{60}$ Participants in Wahbeh's study were consistently medicated for at least 4 weeks before assessment, and a lower peak frequency HF-HRV was observed in individuals with PTSD than in healthy controls. ${ }^{32}$ There is evidence 
Table 2. Meta-regression analyses of the effects of potential variables

\begin{tabular}{|c|c|c|c|c|c|c|c|c|c|c|}
\hline \multirow{2}{*}{ Variable } & \multicolumn{5}{|c|}{ HF-HRV } & \multicolumn{5}{|c|}{ RMSSD } \\
\hline & $\mathrm{N}$ & Coefficient & SE & $\mathrm{Z}$ & $\mathrm{P}$ & $\mathrm{N}$ & Coefficient & SE & $\mathrm{Z}$ & $\mathrm{P}$ \\
\hline Year & 15 & $-0.109(-0.272,-0.055)$ & 0.084 & -1.30 & 0.194 & 10 & $-0.281(-0.697,0.135)$ & 0.212 & -1.32 & 0.186 \\
\hline Quality & 15 & $0.692(-0.067,1.451)$ & 0.387 & 1.79 & 0.074 & 10 & $0.374(-0.515,1.263)$ & 0.453 & 0.83 & 0.409 \\
\hline Age & 13 & $-0.080(-0.198,0.038)$ & 0.060 & -1.33 & 0.184 & & & & & \\
\hline BDI & 10 & $0.016(-0.186,0.218)$ & 0.103 & 0.16 & 0.874 & & & & & \\
\hline Proportion of male participants & 13 & $-0.005(-0.037,0.028)$ & 164.4 & 1.30 & 0.195 & 10 & $-0.019(-0.054,0.016)$ & 0.018 & -1.07 & 0.187 \\
\hline
\end{tabular}

Stata 15.0 was used for meta-regression. HF-HRV: high-frequency heart rate variability, RMSSD: root mean square of successive R-R interval differences, BDI: Beck Depression Scale

Table 3. Subgroup analyses of the effects of potential variables

\begin{tabular}{|c|c|c|c|c|c|c|c|c|}
\hline & \multicolumn{4}{|c|}{ HF-HRV } & \multicolumn{4}{|c|}{ RMSSD-HRV } \\
\hline & $\mathrm{N}$ & $\mathrm{Z}$ & $\mathrm{g}(95 \% \mathrm{CI})$ & $\mathrm{p}$ & $\mathrm{K}$ & $\mathrm{Z}$ & $\mathrm{g}(95 \% \mathrm{CI})$ & $\mathrm{p}$ \\
\hline \multicolumn{9}{|l|}{$\overline{\text { Country }}$} \\
\hline Asia & 7 & 3.27 & $-2.17(-3.47,-0.87)$ & 0.0001 & 3 & 2.27 & $-2.62(-4.88,-0.36)$ & 0.0200 \\
\hline Europe & 3 & 2.08 & $-1.37(-2.66,-0.08)$ & 0.0400 & 3 & 3.02 & $-2.50(-4.11,-0.88)$ & 0.0030 \\
\hline America & 5 & 1.74 & $1.30(-0.16,2.76)$ & 0.0800 & 4 & 4.00 & $-1.00(-1.50,-0.51)$ & 0.0001 \\
\hline \multicolumn{9}{|l|}{ Source of participants } \\
\hline Clinical & 9 & 3.44 & $-1.92(-3.02,-0.83)$ & $<0.00001$ & 7 & 4.08 & $-2.39(-3.53,-1.24)$ & 0.00001 \\
\hline Community & 6 & 1.19 & $0.76(-0.49,2.00)$ & 0.2300 & 1 & 1.73 & $-0.96(-2.05,0.13)$ & 0.0800 \\
\hline Clinical \& Communtiy & 3 & 1.13 & $1.80(-1.31,4.90)$ & 0.2600 & 2 & 5.33 & $-1.03(-1.41,-0.65)$ & 0.00001 \\
\hline \multicolumn{9}{|l|}{ Recording duration } \\
\hline Short-time & 13 & 3.85 & $-1.81(-2.74,-0.89)$ & 0.0001 & 6 & 4.47 & $-2.36(-3.39,-1.32)$ & 0.00001 \\
\hline Long-time & 2 & 1.53 & $-0.27(-0.61,0.07)$ & 0.1300 & 4 & 2.78 & $-1.15(-1.96,-0.34)$ & 0.0050 \\
\hline \multicolumn{9}{|l|}{ Reported values } \\
\hline Raw & 8 & 2.73 & $-1.37(-2.35,-0.39)$ & 0.0060 & & & & \\
\hline Log transformed & 7 & 2.99 & $-1.85(-3.06,-0.64)$ & 0.0030 & & & & \\
\hline
\end{tabular}

Review manager 5.1 was used for subgroup analysis. HF-HRV: high frequency-heart rate variability, RMSSD: root mean square of successive $\mathrm{R}-\mathrm{R}$ interval differences, $\mathrm{CI}$ : confidence interval

that HRV reduction predates the development of PTSD. A large cohort study revealed that decreased HRV at baseline predicted PTSD at follow up, ${ }^{61}$ indicating that HRV alterations might predate the development of PTSD. Therefore, it is possible that disturbance of the ANS may be a biomarker of the susceptibility to PTSD patients, not results.

\section{Clinical implications}

Our results have a wide range of important diagnostic and treatment implications for PTSD. Decreased HRV might be considered as an endophenotype related to increased inflammation, alterations in brain structure and function, impaired executive dysfunction and emotion dysregulation in PTSD. Furthermore, HRV could serve as a valuable biomarker to monitor PTSD and treatment outcomes. An intervention that increases cardiac vagal tone may mitigate the imbalances in PTSD. A few intervention studies have aimed to increase
HRV in PTSD patients via different approaches, with promising results obtained from relaxation training, mindfulness training, ${ }^{62,63}$ and pharmacological treatment. ${ }^{58}$ In addition, individuals who show higher HRV during resting conditions seem to score higher on resilience questionnaires, to recover more efficiently from acute psychological stress and to be less vulnerable to the development of PTSD. ${ }^{65}$ Moreover, HRV reduction may predate PTSD. Therefore, we can intervene in susceptible populations by increasing HRV in advance to decrease the prevalence of PTSD.

\section{Limitations}

This study has several limitations. First, some studies had to be excluded because the results were given in the form of charts and we received no response from the contacted authors. However, all of these excluded studies revealed lower HRV for PTSD than for healthy controls. Thus, their exclusion would not 
change the results. Second, there was not a sufficient number of studies for a meta regression, and possible sources of bias need to be investigated in the future. Subgroup analysis was conducted across trials and not within trials; thus, we can conclude that the studied variables had no influence only at the study level. Third, because most of the articles did not report the trauma type or involved participants who had experienced different traumas, we were unable to explore HRV by trauma type.

We found a reduction in HRV in patients with PTSD relative to HRV in healthy controls, which cannot be completely attributed to medication use. Considering that HRV is potentially associated with central-peripheral integration, changes in inflammatory factors, PTSD-related brain regions and executive function, this appears to be a promising endophenotype for PTSD research.

\section{Acknowledgments}

This study was financially supported by the Special Project on Natural Chronic Non-infectious Diseases (grant nos. 2016YFC1307201); the National Natural Science Foundation of China (Grant Nos. 81701328, 81871061, and 81371484); the National Key Research \& Development Program of China (grant nos. 2016YFC1307200); the China Postdoctoral Science Foundation (grant nos. 2017M612972) and the Postdoctoral Foundation of Sichuan University (grant nos. 2018SCU12042) to Dr. H.Z; and the Department of Science \& Technology of Sichuan Province (grant nos. 2018 SZ0131).

\section{Conflicts of Interest}

The authors have no potential conflicts of interest to disclose.

\section{Author Contributions}

Conceptualization: Fenfen Ge. Data curation: Fenfen Ge, Ying Li. Formal analysis: Fenfen Ge. Funding acquisition: Wei Zhang. Investigation: Minlan Yuan. Methodology: Fenfen Ge. Project administration: Fenfen Ge. Resource: Wei Zhang. Software: Fenfen Ge. Supervision: Wei Zhang. Validation: Wei Zhang. Visualization: Fenfen Ge. Writing-original draft: Fenfen Ge. Writing-review \& editing: Minlan Yuan, Wei Zhang.

\section{ORCID iDs}

$\begin{array}{ll}\text { Wei Zhang } & \text { https://orcid.org/0000-0002-8991-4305 } \\ \text { Fenfen Ge } & \text { https://orcid.org/0000-0003-1516-6698 }\end{array}$

\section{REFERENCES}

1. Kessler RC BP, Demler O, Jin R, Merikangas KR, Walters EE. Lifetime prevalence and age-of-onset distributions of DSM-IV disorders in the National Comorbidity Survey Replication. Arch Gen Psychiatry 2005; 62:593-602.

2. Britvić D AV, Kaliterna M, Lušić L, Beg A, Brajević-Gizdić I, Kudrić M, et al. Comorbidities with posttraumatic stress disorder (PTSD) among combat veterans: 15 years postwar analysis. Int J Clin Health Psychol 2015;15:81-92.

3. Brudey C, Park J, Wiaderkiewicz J, Kobayashi I, Mellman TA, Marvar PJ. Autonomic and inflammatory consequences of posttraumatic stress disorder and the link to cardiovascular disease. Am J Physiol Regul Integr Comp Physiol 2015;309:R315-R321.

4. Guina J, Nahhas RW, Mata N, Farnsworth S. Which posttraumatic stress disorder symptoms, trauma types, and substances correlate with suicide attempts in trauma survivors? Prim Care Companion CNS Disord 2017; 19. pii: 17m02177.

5. Schnurr PP, Lunney CA, Bovin MJ, Marx BP. Clinical. Posttraumatic stress disorder and quality of life: extension of findings to veterans of the wars in Iraq and Afghanistan. Clin Psychol Rev 2009;29:727-735.

6. Jakupcak M, Cook J, Imel Z, Fontana A, Rosenheck R, McFall M. Posttraumatic stress disorder as a risk factor for suicidal ideation in Iraq and Afghanistan War veterans. J Trauma Stress 2009;22:303-306.

7. Lewis C, Raisanen L, Bisson JI, Jones I, Zammit S. Trauma exposure and undetected posttraumatic stress disorder among adults with a mental disorder. Depress Anxiety 2018;35:178-184.

8. D’Andrea W, Sharma R, Zelechoski AD, Spinazzola J. Physical health problems after single trauma exposure: when stress takes root in the body. J Am Psychiatr Nurses Assoc 2011;17:378-392.

9. Ulrich-Lai YM, Herman JP. Neural regulation of endocrine and autonomic stress responses. Nat Rev Neurosci 2009;10:397-409.

10. Sherin JE, Nemeroff CB. Post-traumatic stress disorder: the neurobiological impact of psychological trauma. Dialogues Clin Neurosci 2011; 13:263-278.

11. Kemp AH, Quintana DS. The relationship between mental and physical health: insights from the study of heart rate variability. Int J Psychophysiol 2013;89:288-296.

12. Zygmunt A, Stanczyk J. Methods of evaluation of autonomic nervous system function. Arch Med Sci 2010;6:11-18.

13. Force T. Heart rate variability: standards of measurement, physiological interpretation and clinical use. Task Force of the European Society of Cardiology and the North American Society of Pacing and Electrophysiology. Circulation 1996;93:1043-1065.

14. Pinna GD, Maestri R, Torunski A, Danilowicz-Szymanowicz L, Szwoch M, La Rovere MT, et al. Heart rate variability measures: a fresh look at reliability. Clin Sci (Lond) 2007;113:131-140.

15. Ernst G. Heart-rate variability-more than heart beats? Front Public Health 2017;5:240.

16. Appelhans BM, Luecken LJ. Heart rate variability as an index of regulated emotional responding. Rev Gen Psychol 2006;10:229-240.

17. Beauchaine TP, Thayer JF. Heart rate variability as a transdiagnostic biomarker of psychopathology. Int J Psychophysiol 2015;98:338-350.

18. Berntson GG, Bigger JT Jr, Eckberg DL, Grossman P, Kaufmann PG, Malik M, et al. Heart rate variability: origins, methods, and interpretive caveats. Psychophysiology 1997;34:623-648.

19. Quintana DS, Alvares GA, Heathers JA. Guidelines for Reporting Articles on Psychiatry and Heart rate variability (GRAPH): recommendations to advance research communication. Transl Psychiatry 2016;6:e803.

20. Heathers JA. Everything hertz: methodological issues in short-term frequencydomain HRV. Front Physiol 2014, 5:177.

21. Heart rate variability: standards of measurement, physiological interpretation and clinical use. Task Force of the European Society of Cardiology and the North American Society of Pacing and Electrophysiology. Circulation 1996;93:1043-1065.

22. Berntson GG, Lozano DL, Chen YJ. Filter properties of root mean square successive difference (RMSSD) for heart rate. Psychophysiology 2005;42:246-252.

23. Burg MM, Soufer R. Post-traumatic stress disorder and cardiovascular disease. Curr Cardiol Rep 2016;18:94.

24. La Rovere MT, Pinna GD, Maestri R, Mortara A, Capomolla S, Febo O, et al. Short-term heart rate variability strongly predicts sudden cardiac death in chronic heart failure patients. Circulation 2003;107:565-570.

25. Jovanovic T, Ressler KJ. How the neurocircuitry and genetics of fear inhibition may inform our understanding of PTSD. Am J Psychiatry 2010;167:648-662.

26. Thayer JF, Hansen AL, Saus-Rose E, Johnsen BH. Heart rate variability, prefrontal neural function, and cognitive performance: the neurovisceral integration perspective on self-regulation, adaptation, and health. Ann Behav Med 2009;37:141-153.

27. Thayer JF, Jane RD. A model of neurovisceral integration in emotion 
regulation and dysregulation. J Affect Disord 2000;61:201-216.

28. Thayer JF, Ahs F, Fredrikson M, Sollers JJ 3rd, Wager TD. A metaanalysis of heart rate variability and neuroimaging studies: implications for heart rate variability as a marker of stress and health. Neurosci Biobehav Rev 2012;36:747-756.

29. Hauschildt M, Peters MJ, Moritz S, Jelinek L. Heart rate variability in response to affective scenes in posttraumatic stress disorder. Biol Psychol 2011;88:215-222.

30. Lakusic N, Fuckar K, Mahovic D, Cerovec D, Maisec M, Stancin N. Characteristics of heart rate variability in war veterans with post-traumatic stress disorder after myocardial infarction. Mil Med 2007;172: 1190-1193.

31. Agorastos A, Boel JA, Heppner PS, Hager T, Moeller-Bertram T, Haji U, et al. Diminished vagal activity and blunted diurnal variation of heart rate dynamics in posttraumatic stress disorder. Stress 2013;16: 300-310.

32. Wahbeh H, Oken BS. Peak high-frequency HRV and peak alpha frequency higher in PTSD. Appl Psychophysiol Biofeedback 2013;38:57-69.

33. Cohen H, Benjamin J, Geva AB, Matar MA, Kaplan Z, Kotler M. Autonomic dysregulation in panic disorder and in post-traumatic stress disorder: application of power spectrum analysis of heart rate variability at rest and in response to recollection of trauma or panic attacks. Psychiatry Res 2000;96:1-13.

34. Lee SM, Han H, Jang KI, Huh S, Huh HJ, Joo JY, Chae JH. Heart rate variability associated with posttraumatic stress disorder in victims' families of sewol ferry disaster. Psychiatry Res 2018;259:277-282.

35. Tucker P, Pfefferbaum B, Jeon-Slaughter H, Khan Q, Garton T. Emotional stress and heart rate variability measures associated with cardiovascular risk in relocated Katrina survivors. Psychosom Med 2012;74:160-168.

36. Chang HA, Chang CC, Tzeng NS, Kuo TB, Lu RB, Huang SY. Decreased cardiac vagal control in drug-naive patients with posttraumatic stress disorder. Psychiatry Investig 2013;10:121-130.

37. Moon E, Lee SH, Kim DH, Hwang B. Comparative study of heart rate variability in patients with schizophrenia, bipolar disorder, post-traumatic stress disorder, or major depressive disorder. Clin Psychopharmacol Neurosci 2013;11:137-143.

38. Friedman BH. An autonomic flexibility-neurovisceral integration model of anxiety and cardiac vagal tone. Biol Psychol 2007;74:185-199.

39. Chalmers JA, Quintana DS, Abbott MJ, Kemp AH. Anxiety disorders are associated with reduced heart rate variability: a meta-analysis. Front Psychiatry 2014;5:80.

40. Schmitt L, Regnard J, Desmarets M, Mauny F, Mourot L, Fouillot JP, et al. Fatigue shifts and scatters heart rate variability in elite endurance athletes. PLoS One 2013;8:e71588.

41. Ciccone AB, Siedlik JA, Wecht JM, Deckert JA, Nguyen ND, Weir JP. Reminder: RMSSD and SD1 are identical heart rate variability metrics. Muscle Nerve 2017;56:674-678.

42. Tak LM, Riese H, de Bock GH, Manoharan A, Kok IC, Rosmalen JG. As good as it gets? A meta-analysis and systematic review of methodological quality of heart rate variability studies in functional somatic disorders. Biol Psychol 2009;82:101-110.

43. Higgins JPT, Green S. Cochrane Handbook for Systematic Reviews of Interventions Version 5.1.0 [updated March 2011]. The Cochrane Collaboration 2011. Available at www.handbook.cochrane.org.

44. al Arab AS, Guedon-Moreau L, Ducrocq F, Molenda S, Duhem S, Salleron J, et al. Temporal analysis of heart rate variability as a predictor of post traumatic stress disorder in road traffic accidents survivors. J Psychiatr Res 2012;46:790-796.

45. Higgins JP, Thompson SG. Quantifying heterogeneity in a meta-analysis. Stat Med 2002;21:1539-1558.

46. Ray JM, Pyne JM, Gevirtz RN. Alcohol use disorder moderates the effect of age on heart rate variability in veterans with posttraumatic stress disorder. J Nerv Ment Dis 2017;205:793-800.

47. Shaikh al arab A, Guedon-Moreau L, Ducrocq F, Molenda S, Duhem S, Salleron J, et al. Temporal analysis of heart rate variability as a predic- tor of post traumatic stress disorder in road traffic accidents survivors. J Psychiatr Res 2012;46:790-796.

48. Lee EA, Theus SA. Lower heart rate variability associated with military sexual trauma rape and posttraumatic stress disorder. Biol Res Nurs 2012;14:412-418.

49. Evrengül H, Tanriverdi H, Dursunoglu D, Kaftan A, Kuru O, Unlu U, et al. Time and frequency domain analyses of heart rate variability in patients with epilepsy. Epilepsy Res 2005;63:131-139.

50. Draghici AE, Taylor JA. The physiological basis and measurement of heart rate variability in humans. J Physiol Anthropol 2016;35:22.

51. Souza GG, Mendonca-de-Souza AC, Barros EM, Coutinho EF, Oliveira L, Mendlowicz MV, et al. Resilience and vagal tone predict cardiac recovery from acute social stress. Stress 2007;10:368-374.

52. Williams LM, Das P, Harris AW, Liddell BB, Brammer MJ, Olivieri G, et al. Dysregulation of arousal and amygdala-prefrontal systems in paranoid schizophrenia. Am J Psychiatry 2004;161:480-489.

53. Thayer JF HA, Saus-Rose E, Johnsen BH. Heart rate variability, prefrontal neural function, and cognitive performance: the neurovisceral integration perspective on self-regulation, adaptation, and health. Ann Behav Med Care 2009;37:141-153.

54. Tracey KJ. Reflex control of immunity. Nat Rev Immunol 2009;9:418428.

55. Saeed RW, Varma S, Peng-Nemeroff T, Sherry B, Balakhaneh D, Huston J, et al. Cholinergic stimulation blocks endothelial cell activation and leukocyte recruitment during inflammation. J Exp Med 2005;201: 1113-1123.

56. Passos IC, Vasconcelos-Moreno MP, Costa LG, Kunz M, Brietzke E, Quevedo J, et al. Inflammatory markers in post-traumatic stress disorder: a systematic review, meta-analysis, and meta-regression. Lancet Psychiatry 2015;2:1002-1012.

57. Muscatell KA, Dedovic K, Slavich GM, Jarcho MR, Breen EC, Bower JE, et al. Greater amygdala activity and dorsomedial prefrontal-amygdala coupling are associated with enhanced inflammatory responses to stress. Brain Behav Immun 2015;43:46-53.

58. Cohen H, Kotler M, Matar M, Kaplan Z. Normalization of heart rate variability in post-traumatic stress disorder patients following fluoxetine treatment: preliminary results. Isr Med Assoc J 2000;2:296-301.

59. Kemp AH, Quintana DS, Gray MA, Felmingham KL, Brown K, Gatt JM. Impact of depression and antidepressant treatment on heart rate variability: a review and meta-analysis. Biol Psychiatry 2010;67:10671074.

60. Stein DJ, Ipser J, McAnda N. Pharmacotherapy of posttraumatic stress disorder: a review of meta-analyses and treatment guidelines. CNS Spectr 2009;14:25-31.

61. Minassian A, Maihofer AX, Baker DG, Nievergelt CM, Geyer MA, Risbrough VB, et al. Association of predeployment heart rate variability with risk of postdeployment posttraumatic stress disorder in activeduty marines. JAMA Psychiatry 2015;72:979-986.

62. Lewis GF, Hourani L, Tueller S, Kizakevich P, Bryant S, Weimer B, et al. Relaxation training assisted by heart rate variability biofeedback: Implication for a military predeployment stress inoculation protocol. Psychophysiology 2015;52:1167-1174.

63. Wahbeh H, Goodrich E, Goy E, Oken BS. Mechanistic pathways of mindfulness meditation in combat veterans with posttraumatic stress disorder. J Clin Psychol 2016;72:365-383.

64. Cohen H, Kotler M, Matar MA, Kaplan Z, Loewenthal U, Miodownik $\mathrm{H}$, et al. Analysis of heart rate variability in posttraumatic stress disorder patients in response to a trauma-related reminder. Biol Psychiatry 1998;44:1054-1059.

65. Carnevali L, Koenig J, Sgoifo A, Ottaviani C. Autonomic and brain morphological predictors of stress resilience. Front Neurosci 2018;12:228.

66. Blechert J, Michael T, Grossman P, Lajtman M, Wilhelm FH. Autonomic and respiratory characteristics of posttraumatic stress disorder and panic disorder. Psychosom Med 2007;69:935-943

67. Keary TA, Hughes JW, Palmieri PA. Women with posttraumatic stress 
disorder have larger decreases in heart rate variability during stress tasks. Int J Psychophysiol 2009;73:257-264.

68. Tan G, Dao TK, Farmer L, Sutherland RJ, Gevirtz R. Heart rate variability (HRV) and posttraumatic stress disorder (PTSD): a pilot study. Appl Psychophysiol Biofeedback 2011;36:27-35.

69. Slewa-Younan S, Chippendale K, Heriseanu A, Lujic S, Atto J, Raphael B. Measures of psychophysiological arousal among resettled traumatized Iraqi refugees seeking psychological treatment. J Trauma Stress 2012;25:348-352.

70. Thome J, Densmore M, Frewen PA, McKinnon MC, Theberge J, Nicholson AA, et al. Desynchronization of autonomic response and central autonomic network connectivity in posttraumatic stress disorder. Human
Brain Mapp 2017;38:27-40.

71. Park JE, Lee JY, Kang SH, Choi JH, Kim TY, So HS, et al. Heart rate variability of chronic posttraumatic stress disorder in the Korean veterans. Psychiatry Res 2017;255:72-77.

72. Shah AJ, Lampert R, Goldberg J, Veledar E, Bremner JD, Vaccarino V. Posttraumatic stress disorder and impaired autonomic modulation in male twins. Biol Psychiatry 2013;73:1103-1110.

73. Meyer PW, Müller LE, Zastrow A, Schmidinger I, Bohus M, Herpertz SC, et al. Heart rate variability in patients with post-traumatic stress disorder or borderline personality disorder: relationship to early life maltreatment. J Neural Transm (Vienna) 2016;123:1107-1118. 